\title{
mRNA Vaccine Review against COVID-19 with Potential Applications to Improve Efficacy and Targeting
}

\author{
Mohammed Shahrukh Iqbal, and Shaker A Mousa* \\ Albany College of Pharmacy and Health Sciences, New York, USA
}

*Corresponding author: Shaker A Mousa, PhD, MBA, FACC, FACB, The Pharmaceutical Research Institute, Albany College of Pharmacy and Health Sciences, 1 Discovery Drive, Rensselaer, NY 12144, USA, ORCID: 0000-0002-9294-015X; Tel: 01518 694 7397; Email: shaker.mousa@acphs.edu

Received: 30 Mar, 2021 | Accepted: 21 Apr, 2021 | Published: 27 Apr, 2021

Citation: Iqbal MS, Mousa SA (2021) mRNA Vaccine Review against COVID-19 with Potential Applications to Improve Efficacy and Targeting. Int J Vaccine Immunizat 5(1): dx.doi.org/10.16966/2470-9948.124

Copyright: (C) 2021 lqbal MS, et al. This is an open-access article distributed under the terms of the Creative Commons Attribution License, which permits unrestricted use, distribution, and reproduction in any medium, provided the original author and source are credited.

\begin{abstract}
Coronavirus disease 19 (COVID-19) is a respiratory illness caused by severe acute respiratory syndrome coronavirus 2 (SARS-CoV-2). The virus is the cause of the pandemic that has affected over 213 countries and there are over 21 million confirmed cases in the United States. Intense research efforts and funding have been placed into diagnostic, prevention, and treatment options. This review evaluates mRNA vaccines, their advantages, limitations, and current progress towards vaccine production. We included current methods of mRNA vaccine production and introduce potential changes in vaccine delivery methods currently being developed to specifically aid in targeting the coronavirus. We evaluated various routes of administration and their potential to allow vaccines to reach the immune system and induce an immune response to produce antibodies and provide immunity. Safety and efficacy have been evaluated in this review of various mRNA clinical trials that aimed to determine if mRNA vaccines can cause antibody production and the types of adverse events associated with them. Although mRNA vaccines are not perfect in design, the possibilities they offer in advancing vaccine research and fighting other viral infections while improving immunity are worth further research.
\end{abstract}

Keywords: Coronavirus; COVID-19; SARS-CoV; Vaccines; mRNA; DNA; Lipid nanoparticle (LNP), Mucosal; Intranasal; Pulmonary; Moderna

\section{Introduction}

Severe Acute Respiratory Syndrome Coronavirus (SARS-CoV-2) is a derivative of the Coronaviridae family [1], associated with Middle East Respiratory Syndrome Coronavirus (MERS-CoV) [2] in causing respiratory-related distress and pulmonary failure. SARS-CoV-2 is genetically closely related to the original SARS-CoV virus that caused the outbreak of SARS in Guangdong province, China in 2003 [3]. As of late May 2020, 80,304 reports of pneumonia related symptoms were prevalent among 34 districts in China with 90,870 confirmed cases worldwide. As of Jan $7^{\text {th }}, 2021$, there were $21,259,997$ cases with 299,904 new cases and 359,849 deaths in the US [4]. The source of infection was reported to derive from the consumption of bat and other potentially infected animals that were sold in the Wuhan district local market [5].

Amongst the infected individuals during the Wuhan outbreak, samples from seven patients with severe pneumonia were admitted into the Wuhan Jin-Yin-Tan Hospital ICU unit with the diagnosis of the causative pathogen at the Wuhan Institute of Virology (WIV). 5 samples were found to be PCR-positive for CoVs. Genome sequencing was performed on some sample collected from the bronchoalveolar lavage fluid (BALF), with an $87.1 \%$ similarity to the sequences of
SARSr-CoV. Further analysis was done on the virus of genome consisting of six major reading frames that are like coronavirus. Additionally, a short region of RNA-dependent RNA polymerase from bat coronavirus was found to have high sequence identity to 2019-nCoV, which supports the origin of the 2019-nCoV in bats. Serology studies were per-formed using ELISA assay for IgG and IgM antigen from bat protein to identify the $\mathrm{N}$ protein of the 2019-nCoV, in which $\operatorname{IgG}$ and IgM titers increased over time in the 5 samples. Virus specific nucleotide positivity and serology within these samples provides evidence of the connection between the bat origins during the Wuhan outbreak and its connection to 2019-nCoV. While the transmission of SARSr-CoV still remains unclear, the infection rates between human transmissions rapidly increased via airborne route.

As a result, close contact with others and exposure to any form of coughing, sneezing, talking, respiratory drops, or aerosols could result in the virus accessing the airways of a healthy individual and infecting them [6]. The disease caused by the virus has been named COVID-19 and it is currently infecting people in multiple countries [7]. This has resulted in worldwide quarantine, wearing of masks, social distancing, and other methods to reduce close contact with others until a viable treatment option has been determined $[8,9]$. 
This virus has resulted in the need for a vaccine to be developed to promote immunity and for clinical trials of researched treatment options such as convalescent plasma transfusion [10-12], antivirals, and anti-inflammatory drugs. Current mainstream interest in vaccine development for COVID-19 is focused on mRNA vaccines. This review provides a comprehensive analysis of vaccine production, comparison of current vaccines and a broad review of mRNA vaccines and the potential they have as a vaccine form against COVID-19. Reviewed here are the methodology behind mRNA vaccines and application of current research for pulmonary and mucosal routes of administration. These alternative routes could enhance the specificity of the vaccine against the virus and improve the vaccine production process towards the goal of perfecting and innovating the concept.

The mechanism of coronavirus of how the virus enters and infects cells and the receptor targets has been further identified $[1,13,14]$. The virus utilizes an open reading frame along the downstream area of its nucleotide sequence that encodes for the viral protein and causes the cells to replicate this viral protein like other RNA-based viruses. However, the distinguishing feature of coronavirus is the specific spike (S) proteins present on the surface of the virus that greatly enhance its ability to attach and penetrate the cell [15]. It has been noted in recent studies that SARS-CoV-2 has variations in its amino acid sequences of $8 \mathrm{~b}$ and $3 \mathrm{c}$ and is missing the $8 \mathrm{a}$ protein, further aiding in development of diagnosis methods to determine the presence of COVID-19 in exposed patients [16]. The entry methods of the virus were determined to be specifically through the angiotensin-converting enzyme 2 (ACE2) receptors and are mediated by proteolytic processing (Cath and TMPRSS2) [17-22].

\section{mRNA Vaccines}

mRNA vaccines use specific viral nucleotide sequences, without using the live virus, and try to incorporate it into the host cell to mimic a native response [23]. They do not enter the nucleus of the cell and so do not affect the DNA of the host cell, and the viral mRNA is broken down after all the mRNA sequence has been made [24]. Currently there are six prophylactic mRNA vaccines in phase 1 clinical trials
[25], involved with tumor [26-28] and cardiovascular issue treatments [29-31], but none involved with respiratory-related infections. mRNA focuses primarily on affecting the cell cytoplasm to produce the viral RNA proteins on the surface of the cell for the antigens to be made. Certain limitations include the field actively being researched for specific tissue targeting and requiring immunogenicity response monitoring. mRNA vaccines can overcome many of the problems that plague traditional vaccines and can help with combating against viral infections as highlighted in figures 1 and 2 [32].

mRNA vaccines are a concept that has been introduced and researched extensively since 1995 by trying to induce adaptive immune response in cancer patients to create antitumor immunity $[26,33]$. Due to the potential of creating natural-borne antigens, lack of needing additional adjuvants, and more rapid preparation compared to traditional vaccines while avoiding risk of permanent DNA integration from DNA vaccines, mRNA vaccines have great potential to improve vaccination production and efficacy [34-36].

\section{Function}

mRNA vaccines focus on the incorporation of the viral nucleotide and allow it to enter the cytoplasm of the host cells to produce viral proteins like the virus itself. This allows a similar "native" infection to occur, but at a controlled level, to allow the host's immune system to react. As a result, the antigen is introduced to the surface of the cell, which allows the immune system to respond. CD4 and CD8 will then be recruited to target these specific cells and eradicate them from the body and help in the formation of antibodies against the virus, and memory $\mathrm{B}$ cells will then remember pathogen and aid in faster antibody production [37]. SARS-CoV-2 contains 4 major structural proteins, namely spike $(\mathrm{S})$, membrane $(\mathrm{M})$, and envelope (E) proteins (all of which are embedded in the viral surface envelope), and nucleocapsid nucleoprotein $(\mathrm{N})$ protein. Several of these viral proteins potentially serve as targets of vaccine induced immune responses. However, $S$ proteins are responsible for recognition of the host cellular receptor to initiate viral entry. Available mRNA vaccines work via the

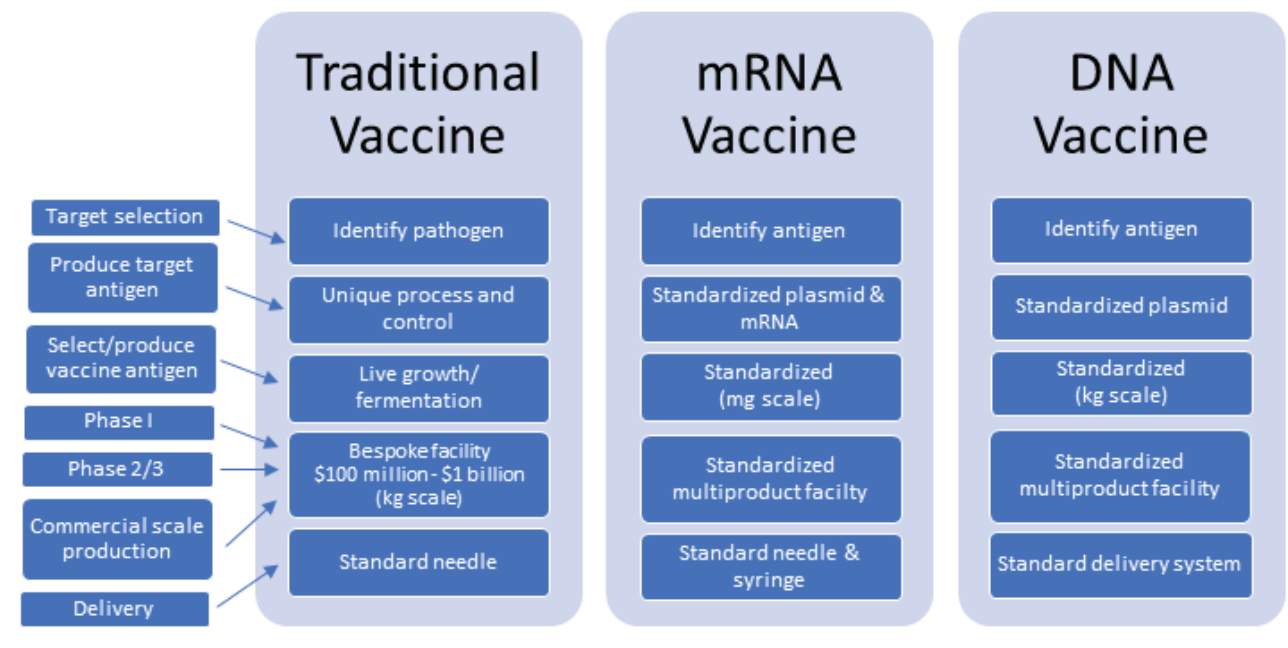

Figure 1: Highlights of the various advantages that mRNA has over traditional vaccine development. Left side lists the sequential parts of vaccine production and development to provide comparison between the three vaccine types. 


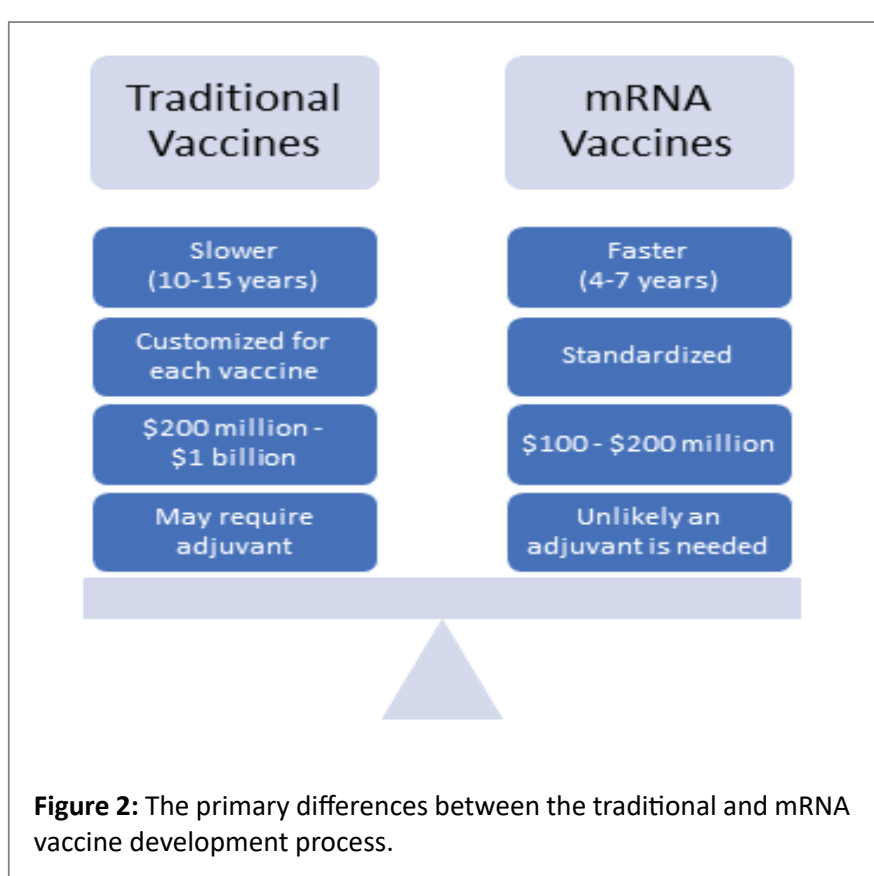

induction of an antibody against the viral surface protein responsible for entry into the human host cells' "spike protein".

The main factors involved with mRNA therapeutic applications include: (1) mRNA 5' and 3' untranslated region (UTR), (2) modification of rare codons for amino acid synthesis to translate into protein production, and (3) inhibition of RNA de-capping and improvement in enzyme degradation resistance. However, introduction of viral protein into the cell includes encapsulating the mRNA in a shell, which allows it to penetrate the cell layer, pass through the various extracellular barriers and be endocytosed into the cytoplasm to be properly translated into proteins by ribosomes (Figure 3). mRNA vaccines and their delivery methods that permeate the lungs for virus targeting could help specifically target and eradicate the virus and possibly resolve multiple respiratory-related symptoms. Current mRNA vaccines against coronavirus focus on targeting the $S$ protein, which allows it to bind to cells effectively to replicate viral proteins [38].

\section{Delivery methods}

The delivery of the mRNA is a crucial step in the process of developing an mRNA vaccine because there are multiple barriers that help prevent infection naturally. The main barriers are categorized into extracellular and intracellular barriers. Extracellular barriers include rapid clearance, nucleases, and localization, and intracellular barriers include endosomal escape, RNA sensors, and endonucleases [39]. Current limitations include that various forms of enzyme degradation are present and optimizing delivery of the mRNA to be accepted by the cell without being rejected by the immune system [40]. Lipid nanoparticles (LNP) are the one of the most studied methods that involves the use of: (1) an ionizable lipid layer that contains aminebearing viral vectors, (2) a zwitterionic lipid, (3) cholesterol molecule, and (4) a polyethylene glycol lipid with helper lipid, all aiding in mRNA viral transmission [41]. Examples of various systems used in mRNA delivery are shown in figure 4 .

The incorporation of an amine group as a non-viral vector aids in formation of the protective layer surrounding the mRNA to help prevent exposure to nucleases and other enzyme degradation processes. An ionizable cation lipid helps regulate the $\mathrm{pH}$ to a level desired for permeation and reduced degradation of the enzyme. A zwitterionic lipid bears a similarity to lipids found in the cell membrane and this helps to prevent a response that results in rejection of the shell. The use of the cholesterol molecule helps to stabilize the overall polymer and the polyethylene glycol lipid to provide a hydrating layer to deliver more stability (Figure 5). All these components work together to help introduce the mRNA into the cytoplasm of the cell via endocytosis while actively disrupting the nucleosomes until the final step where the mRNA is released and then taken by a ribosome to make viral protein [42-44]. Current examples of mRNA vaccines going through clinical trials include Moderna's Cytomegalovirus (CVM) [45,46]. Refer to (Figure 5) for the different categories of delivery systems used to introduce mRNA into the cell and for examples.

\section{Subtypes}

Currently two subtypes of mRNA vaccines have been classified: Non-amplifying mRNA based vaccines (NAM), and self-replication mRNA vaccines (SAM).

NAM vaccines use the base concept of containing a viral nucleotide with an open reading frame to promote translation of specific viral proteins with additional benefits. These benefits include: (1) relatively small size of mRNA needed, (2) absence of additional protein production to lessen potential immunogenic responses, (3) relatively easy to scale up and manufacture in vaccine production and during times of sudden pandemics, and (4) easier to improve the system and modify it to handled new cases of new viral strands that emerge or other viruses with similar genetic codes. However, their main limitation derives from intracellular delivery, which is currently being improved with the introduction and innovation of Lipid Nanoparticles (LNPs). Further research is needed on not only improving the delivery of NAMs but on LNPs as well because they have a limitation of being affected by exocytosis of the cell, making introduction of the mRNA more difficult.

SAM differs from non-amplifying because it involves an mRNA engineered from a positive single-stranded virus like alphaviruses and flaviviruses. These results in two open reading frames that serve two different functions that help form the viral protein when it is being translated. One part helps with RNA capping and promoting replication of the viral nucleotide and the other part helps to express the antigen. Using this method can extend the duration of viral protein translation and stability for up to two months and can increase the magnitude of expression significantly. However, limitations are that nucleotide sequences cannot handle much modification and so it is not versatile, the replicon size is very large to account for the two sections to aid in viral protein replication, and inclusion of potential unrelated protein responses due to the increased amount of replicon available in this method [47-50].

\section{Vaccine Administration Routes}

The main routes of vaccine administration and most common forms are subcutaneous and intramuscularly. Currently two of the FDA Emergency Authorized Use approved vaccines against COVID-19 are administered intramuscularly. Both Moderna and Pfizer vaccines require injection of $0.5 \mathrm{~mL}$ of the vaccine into the patient's deltoid muscle using a 23-25 gauge needle. However, with specific tissue targeting and the COVID-19 pandemic related to respiratory infection, alternative methods of administration have been explored. Current areas of interest, in relation to the coronavirus outbreak include mucosal and intranasal vaccination [51]. 


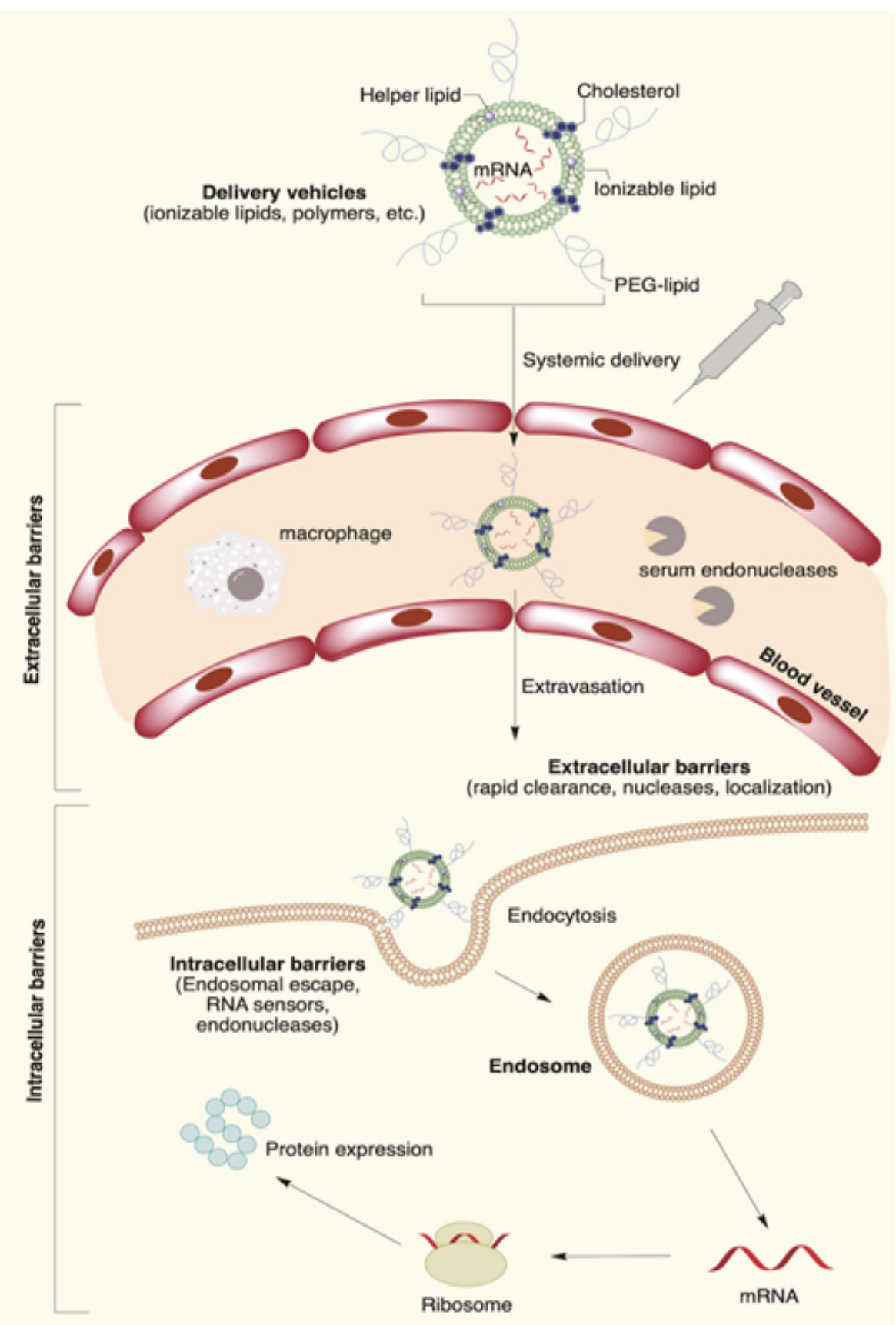

Figure 3: mRNA nucleotide sequences encapsulated in a nano lipid-particle (LNP) formulation entering the cytoplasm of a cell via endocytosis. The endosome is created and then degraded by enzymes to release the mRNA sequence. The mRNA sequence is translated into an amino acid sequence by a ribosome complex and becomes a viral protein sequence that is introduced to the surface of the host cell to be introduced to an antibody [39] and is used under a Creative Commons CC-BY-NC-ND license.

Mucosal vaccination is a promising application that involves activation of several aspects of the immune system in the fastest manner possible with the least amount of side effects [52,53]. This route utilizes the Mucosa-Associated Lymphoid Tissue (MALT) system [54] and Nasopharynx-Associated Lymphoid Tissue (NALT) system to introduce the mRNA to the dendritic cells of the immune system to cause a humoral immune response. This allows the mRNA to access the specific components of the immune system of the lungs that include alveolar macrophages, dendritic cells, $M$ cells, and intraepithelial cells. The idea is to expose the mRNA to dendritic cells to cause a humoral response $[55,56]$. Intranasal vaccination is another promising application that focuses on formulating the vaccine in small particles taken in by the nasal route to reach the lungs specifically [57].

Particle size, shape modulation, charge manipulation and variation of the antigen to be properly taken up by antigen-presenting cells are 


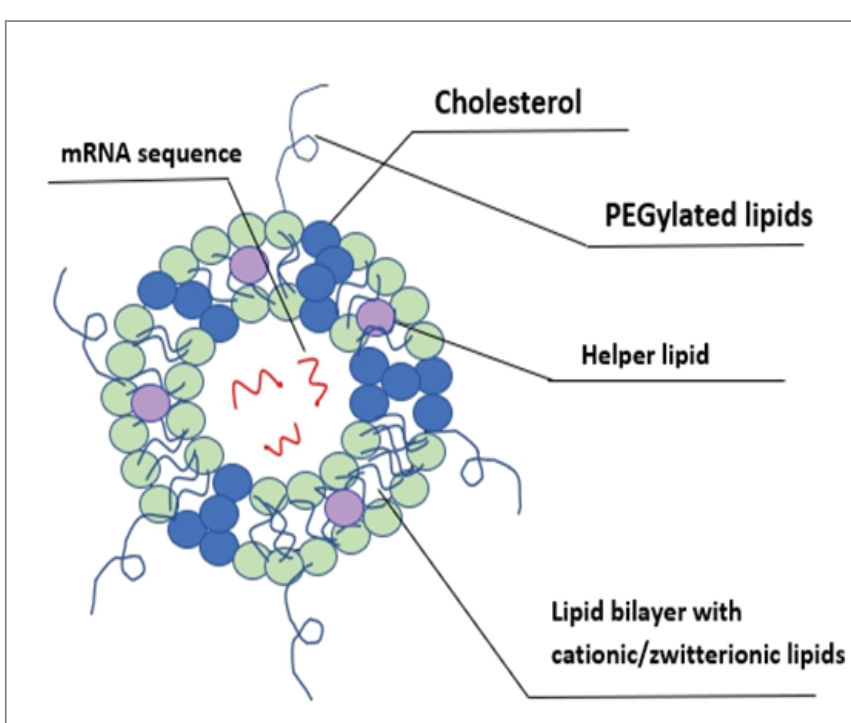

Figure 4: Diagram of an mRNA sequence within a lipid nanoparticle (LNP) delivery vehicle.

areas of study necessary to ensure that the vaccine can be introduced via mucosal route. Current research has determined that particle size should be less than 3 micrometers, with best results for dendritic celltargeted uptake of particle size less than 0.5 micrometers [58]. Regarding particle shape, a study from LIQUIDIA found that when earthworms' immune systems were introduced to variously shaped antigen particles, antibody titers were the highest amongst the Particle Replication in Non-Wetting templates (PRINT) particles [59,60]. PRINT particles are a continuous, wetting, roll-to-roll molding technology that creates microparticles and nanoparticles and allows them to be adjusted to attain specific parameters associated with enhancing their function and stability. Manipulating charge and increasing stability will further enhance the ability of the vaccine to reach dendritic cells because positive charge results in better interaction with cell membranes to enhance permeation ability. PEGylation, dry spray, dry freezing spray and use of stabilizers such as lectin, mannitol, dextran, or sorbitol are used to further enhance and stabilize the complex to enter the mucosa via inhalation route more easily [61].

Pulmonary-targeted vaccines are another area of interest as a possible alternative because they utilize a dry powder or a liquid formulation [62]. A current example of pulmonary vaccination in development is for measles vaccines [63]. These vaccines are put into a metered dose inhaler or nebulizer to effectively reach the alveoli of the lungs to then be taken in and introduced to the immune system to cause a response. For optimal pulmonary delivery, a particle size between 0.5 and 5 micrometers is optimum, and the characteristics of dispersion type, device used and dispersion in the airstream can affect the mass median aerodynamic diameter [64]. All these factors impact the overall ability of the particle to deliver the vaccine to the lungs. Particle size has an additional effect because it impacts various regions of the lungs for pathogen targeting. Particle sizes larger than 5 micrometers are optimal against pathogens stationed in the upper respiratory tract, whereas particle sizes less than 3 micrometers are optimal for lower and deep respiratory tract infection. However, regarding formulation, there are limited options for excipients, and they must meet stability and safety standards like those specified for common inhalation products. The nanoparticle delivery system is affected by particle size and polymorphic changes in the matrix and the structure is further evaluated by X-ray refraction because morphology of the particle will affect its efficacy in penetration $[60,65]$. The development process of inhaled vaccines is highlighted in (Figure 6).

\section{Safety and Efficacy mRNA vaccines}

mRNA vaccines are currently still under development and issues of potency and toxicity are areas of concern. Inflammatory responses are a common side effect of general vaccinations indirectly, but with the inclusion of mRNA vaccines directly affecting the TLR 3,7,8 and cytoplasmic pathways, the response is potentially more potent. Introduction of a viral nucleotide to induce a humoral response in the immune system results in potential immunogenic responses. These responses caused adverse events in clinical trials for antivirals and anticancer drugs resulting in nerve damage, myopathy, and pancreatitis [66]. Liver toxicity is another potential area of concern as found in a preclinical study using an LNP formulation. The combination of the multiple dosing and the formulation were causes for the toxicity and could serve as references for future LNP formulation and vaccine development. Multiple dose administrations are needed for mRNA vaccines to produce the viral protein to properly elicit the immune response, which contributes to the potential of adverse events occurring and could inversely impact the translation of the mRNA. mRNA vaccines have a small, but less likely, potential of permanent integration with the host DNA compared to their DNA vaccine counterparts, and this remains a potential safety concern. Other issues include the potential to cause autoimmunity or worsening other autoimmune-related disease states like diabetes mellitus and multiple sclerosis. Further research is needed to evaluate and reduce these adverse events while enhancing the benefits of mRNA vaccines.

\section{Moderna RSV mRNA vaccine}

Respiratory syncytial virus (RSV) is a virus that causes upper and lower respiratory tract infection. A vaccine targeting the RSV F protein is an attractive target for vaccine development, and Moderna has developed an RSV vaccine using a modified RSV F mRNA sequence with an LNP base. The study was conducted on rodents, and overall efficacy regarding an immunogenic response and protection formed were observed. Using ELISA titers of the transfected cells revealed secretion and stabilization of the antibodies formed. The mice were injected with 2 doses of 10 micrograms intramuscularly 3 weeks apart, with serums collected 2 weeks after. Results suggest that with the LNP formulation, the RSV F protein elicited a humoral response of the immune system in mice. Higher CD4+ and CD8+ T cell responses were observed in mice, but challenges of human vaccination include the potential for vaccine developed respiratory disease (VERD), possibly leading to increased hospitalization and death if used in naïve human infants. Further research is needed to improve the LNP delivery system and to reduce adverse events in future human vaccination [67].

\section{H10N8 and H7N9 mRNA vaccine}

The H10N8 (Germany) and H7N9 (USA) vaccines were mRNAbased vaccines against influenza [68]. Both were randomized, placebocontrolled trials that involved administration of 2 doses of the vaccines over a 3-week period to assess overall safety and efficacy. The H10N8 trial was in Berlin, Germany, and the H7N9 vaccine trial was in Miami, Florida. H10N8 participants were between the ages of 18 to 64 years old and received doses of 25, 50, 75, 100 and 400 micrograms intramuscularly and 25 and 50 micrograms intradermally. H7N9 participants were adults between the ages of 18 to 49 years old and 


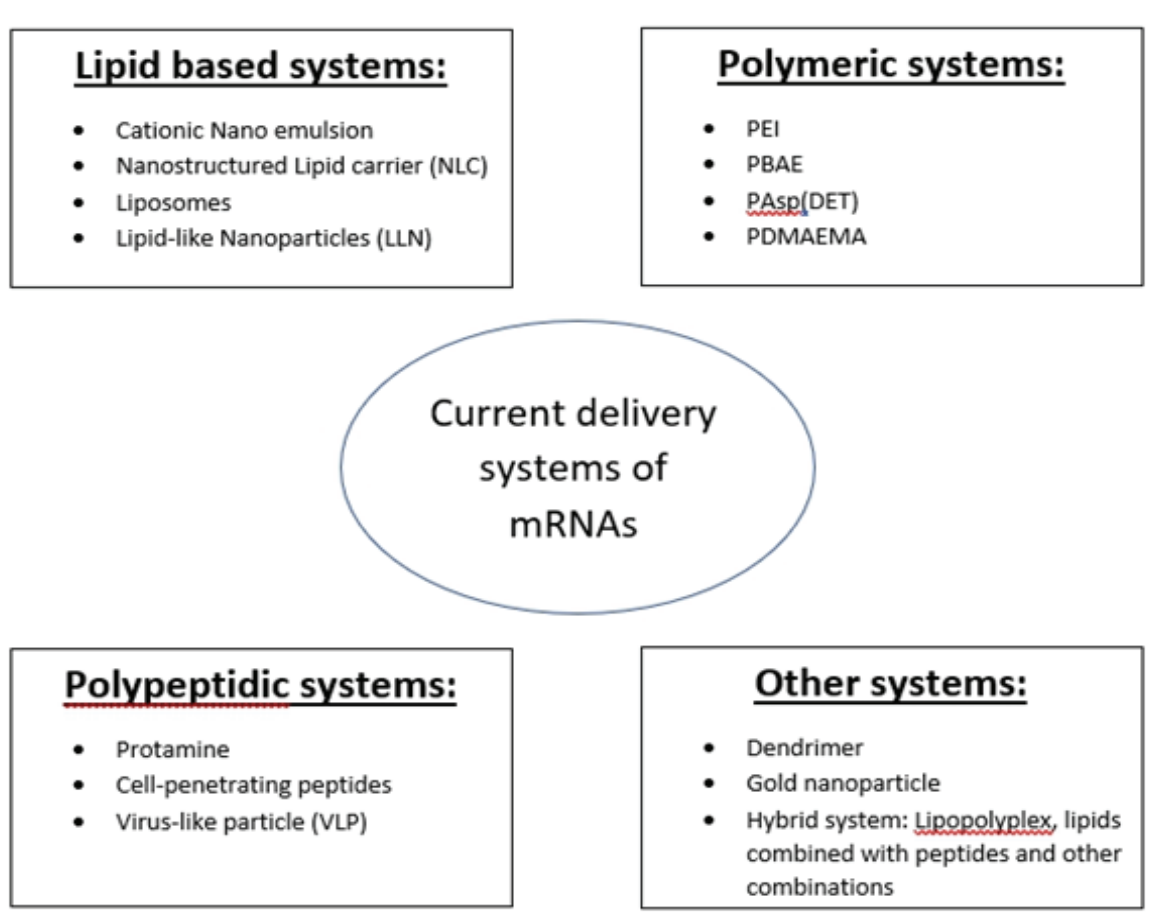

Figure 5: Current systems for mRNA delivery.

Abbreviations: PEI, polyethylenei-mines; PBAE, poly (beta-amino esters); PAsp, poly(asparmatides); DET: 1,2-diaminoethane addition; PDMAEMA, poly (2-dimethylaminoethyl methacrylate.

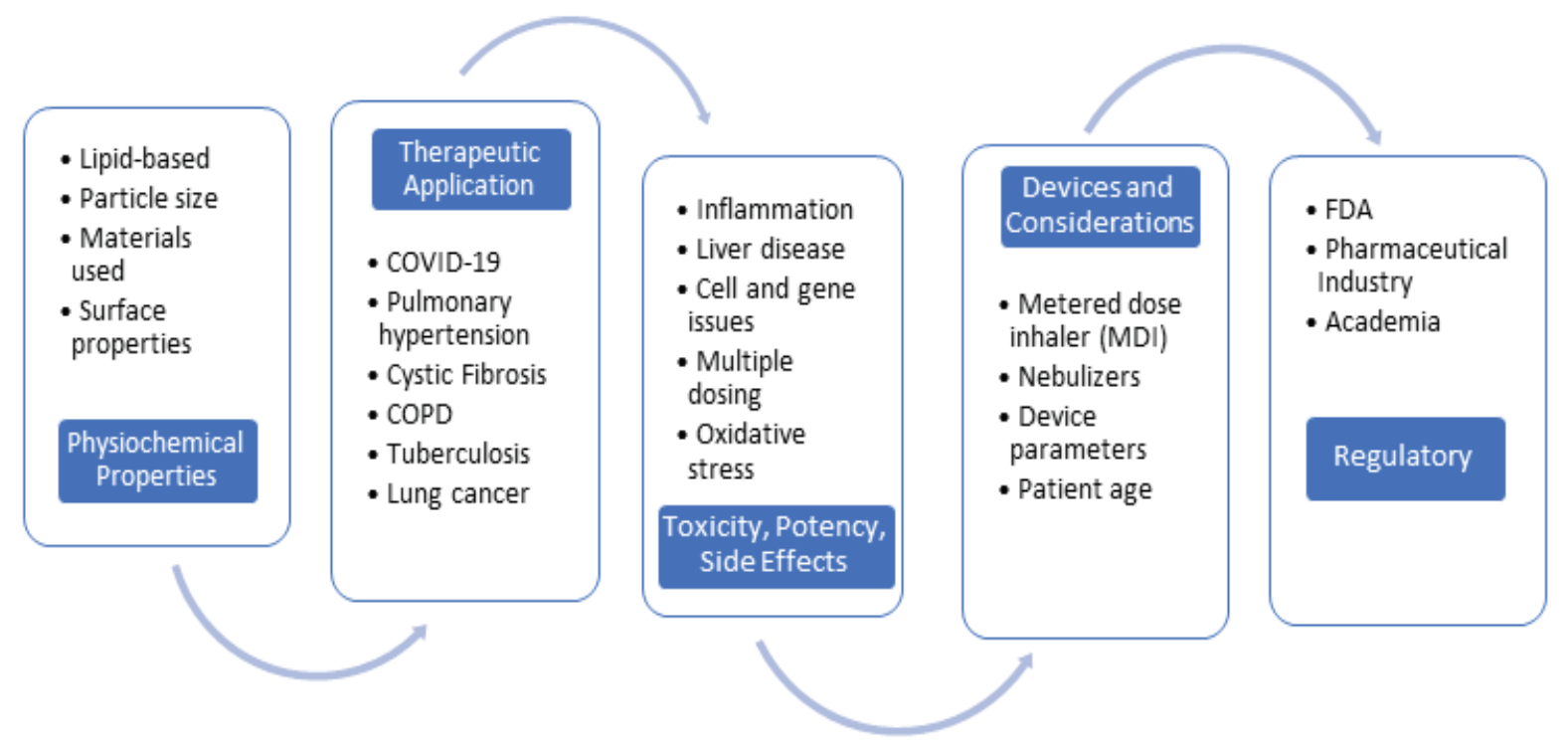

Figure 6: Diagram of future obstacles, research, and development processes for respiratory vaccines categorized into targeted areas based on figure 1 [62] and used under a Creative Commons Attribution license. 
received intramuscular doses of 10,25 , and 50 micrograms with the 25 and 50 microgram groups able to receive a booster shot at 6 months. Safety was assessed through screenings of physical examination and vital signs at days $1,22,30$, and 43 and type of adverse events were graded according to severity between 1 (mild) and 4 (life threatening) [68]. Immunogenicity was measured using antibody serum detection, titers, and hemagglutination inhibition assays. Results of both studies showed favorable safety profiles with no vaccine-related adverse events reported. Both studies showed that the higher doses of the intramuscular injection resulted in greater microneutralization assay titer formation with no significant cell-mediated response noticed. These titers are indicative for antibody formation against influenza specifically. Further evaluation is needed for future perspectives of vaccine development $[68,69]$.

\section{SARS-CoV-2 vaccines}

Table 1 presents some vaccines currently in clinical trials for SARCoV-2.

Moderna mRNA-1273: Moderna conducted a randomized, 1:1 placebo-controlled study to test mRNA-1273 at the $100 \mathrm{mcg}$ dose level in 30,000 participants ages 18 and older in the US. The primary end-point was the prevention of symptomatic COVID-19 disease based on analysis of COVID-19 cases confirmed and consolidated starting 2 weeks after the second dose of vaccine. Secondary endpoints included prevention of severe COVID-19 disease and prevention of infection by SARS-CoV-2. This study included more than 7,000 people age 65 and over and more than 5,000 people under age 65 who have high-risk chronic disease (severe obesity, diabetes, cardiac disease; $42 \%$ of the study participants). It also included 11,000 participants from communities of color (Hispanic or Latina and Black or African American; $37 \%$ of the study population). The study determined that a total of 151 cases would provide $90 \%$ power to detect a $60 \%$ reduction in COVID-19 hazard rates. Interim analysis included 95 participants with confirmed COVID-19: 90 cases in the placebo group and 5 cases in the mRNA-1273 group for a vaccine efficacy of $94.5 \%(\mathrm{P}<0.0001)$. Among the placebo group, 15 cases were present in the adults age 65 and over and there were 30 severe cases overall. The vaccine was generally well tolerated with most adverse events being mild to moderate in severity. Grade 3 (severe) events were greater than or equal to $2 \%$ in frequency and were identified as respiratory rate of 30 breaths per minute or higher, heart rate exceeding 125 beats, respiratory failure, renal failure, hepatic impairment, admission to an intensive care unit, or death. Adverse events after the first dose included injection site pain $(2.7 \%)$ and after the second dose included fatigue $(9.7 \%)$, myalgia (8.9\%), arthralgia (5.2\%), headache (4.5\%), pain (4.1\%), and redness at injection site (2.0\%). These events occurred about 15 hours after vaccination and were typically resolved by day 2 . Initial analysis suggested a broadly consistent safety and efficacy profile across all evaluated subgroups [79].

Pfizer BNT162b2 SARS-CoV-2 vaccine: In the Pfizer trial of BNT162b2 conducted between July 27 and November 14, 2020, there were 43,548 participants age 16 years or older at 152 sites worldwide (the majority of the sites were in the US). Participants were randomly assigned in a 1:1 ratio to receive $30 \mathrm{mcg}$ of BNT162b2 $(0.3 \mathrm{~mL}$ volume per dose) in 2 doses administered intramuscularly, 21 days apart, or saline placebo delivered in the deltoid muscle. Inclusion criteria were age 16 years or older who were healthy or had a stable chronic medical condition, including but not limited to HIV, hepatitis B, or hepatitis $\mathrm{C}$ infection. Exclusion criteria were a medical history of COVID-19, treatment with immunosuppressive therapy, or diagnosis with an immunocompromising condition. Major primary endpoints included efficacy of BNT162b2 against confirmed COVID-19 (onset present up to 7 days after the second dose), efficacy with and without evidence of prior infection, solicited and unsolicited adverse event recording (throughout the following 6 months after the second dose) and use of antipyretics or pain medications (within 7 days after each dose). Current adverse event monitoring data represents the 14 days after second dose administration, and HIV safety results were to be included in a separate report. Safety monitoring will continue for 2 years after administration of the second dose of the vaccine. Major secondary endpoints included efficacy of BNT162b2 against severe COVID-19 (respiratory failure, severe systemic illness, evidence of shock, significant acute renal, hepatic, or neurological dysfunction, admission to an ICU, or death). Daily use of the electronic diary ranged

Table 1: Current COVID-19 vaccine production listed by company and type of vaccine.

\begin{tabular}{|c|c|c|c|}
\hline Company & Vaccine Type & Current Progress & Reference \\
\hline Moderna & mRNA & $\begin{array}{l}\text { Currently vaccinating healthcare } \\
\text { providers, and patients over } 65+\text { of age } \\
\text { and those with underlying comorbidities }\end{array}$ & [70] \\
\hline Inovio Pharmaceuticals & DNA based & Currently in Phase 1 trials & {$[71]$} \\
\hline Oxford University & Adenovirus-vectored & Currently in Phase 1 trials & {$[72]$} \\
\hline $\begin{array}{l}\text { Pfizer Pharmaceuticals/ } \\
\text { BioNTech }\end{array}$ & $\begin{array}{c}\text { Lead vaccine } \\
\text { candidate/mRNA } \\
\text { candidate/self-amplifying } \\
\text { mRNA candidate }\end{array}$ & $\begin{array}{l}\text { Currently vaccinating healthcare } \\
\text { providers, and patients over } 65+\text { of age } \\
\text { and those with underlying comorbidities }\end{array}$ & [73] \\
\hline $\begin{array}{l}\text { Johnson \& Johnson/ } \\
\text { Sanofi }\end{array}$ & Lead vaccine candidate & $\begin{array}{l}\text { Currently gaining Emergency Use } \\
\text { Authorization for vaccination }\end{array}$ & {$[74]$} \\
\hline $\begin{array}{l}\text { University of Queensland } \\
\text { In Australia }\end{array}$ & mRNA & Preclinical trials & [75] \\
\hline Altimmune & Single dose, intranasal & Currently in Phase 1 trials & {$[76]$} \\
\hline Novavax & Lead vaccine candidate & Currently in Phase 1 trials & [77] \\
\hline Vaxart & Oral vaccine candidate & Preclinical trials & [78] \\
\hline
\end{tabular}


from $90-93 \%$ for each day after the first dose and $75-83 \%$ for each day after the second dose (no difference was noted between the groups). No deaths were related to the vaccine and no COVID-19 associated deaths were observed. Among 36,523 participants who had no evidence of existing or prior SARS-CoV-2 infection, 8 cases of COVID-19 with onset at least 7 days after the second dose were observed among the vaccine recipients and 162 cases were observed among the placebo recipients; this corresponded to $95 \%$ vaccine efficacy. Between the first and second dose, 39 COVID-19 cases in the BNT162b2 group and 82 COVID-19 cases in the placebo group were observed, resulting in a vaccine efficacy of $52 \%$ and indicating early protection by the vaccine starting as soon as 12 days after the first dose [80].

\section{Conclusions}

mRNA vaccines provide a potential method to form antibodies particular to the spike protein of the coronavirus and provide specific protection against COVID-19. Further research and observation are needed to show if COVID-19 vaccines will be an effective longterm solution. The concept of vaccinations is involved with the early, middle, and end stages of a pandemic outbreak. Vaccines provide herd immunity and can protect succeeding generations from viral infections and effectively reduce the infection's impact to the point of completely eradicating future infections [81]. Vaccines produce antibody titers formed from introducing a viral antigen to a host immune system and causing a response. These antibodies improve diagnostic methods to identifying infected patients, noninfected patients, or patients who had developed antibodies naturally due to previous exposure or those who are asymptomatic.

Current FDA approval of vaccine use has been granted to both Moderna's mRNA-1273 and Pfizer's BNT162b2 for Emergency Authorized Use (EAU) in hospitals and for first res-ponders above the age of 18. A concern of COVID-19 vaccinations is vaccinating individuals who have developed antibodies naturally due to previous exposure to the virus. This has the potential risk of antibody-mediated disease enhancement when considering increased hyper-immune globulin production in COVID-19 patients. A recent study evaluates this hypothesis and states that while the risks are low, more research is needed to evaluate further and gain a better understanding of clinical evidence for immune enhancements via vaccinations [82].

Vaccine production and innovation will require more time and research to not only improve the concept of a vaccine but to mitigate many of its weaknesses. With mRNA vaccines, the side effects are still being evaluated to learn how to reduce them. Enhancement of the LNP delivery system and culminating the advancements and research involved with pulmonary and mucosal vaccine delivery could help unlock the potential of mRNA vaccine and help it target specifically against COVID-19 and respiratory diseases in general. Other potential applications of vaccinations, such as the Bacillus Calmette-Guerin vaccine, include avoiding COVID-19 complications in those with chronic illnesses like diabetes and potentially applying it to other disease states [83]. With the rapid accumulation of SARS$\mathrm{CoV}-2$, genome sequences of thousands of genomic variants of SARSCoV-2 are now publicly available. mRNA vaccine might provide broad protection against some of the variants, but still more data are needed. Further research is also needed regarding dosage variations, concerning potential inhalation formulations, nasal formulations, development of vaccines against the common variants and other potential short-term and-long term side effects.

\section{Author Contributions}

Conceptualization, M.S.I. and S.A.M.; formal analysis, M.S.I.; resources, S.A.M.; writing original draft preparation, M.S.I.; writing review and editing, M.S.I. and S.A.M; supervision, S.A.M. All authors have read and agreed to the published version of the manuscript.

\section{Institutional Review Board Statement}

Not applicable

\section{Informed Consent Statement}

Not applicable

\section{Data Availability Statement}

Not applicable

\section{Declaration of Competing Interest}

The authors of this manuscript declare no conflict of interest.

\section{Funding}

This research did not receive any specific grant from funding agencies in the public, commercial, or not-for-profit sectors.

\section{References}

1. Koichi Y, Miho F, Sophia K (2020) COVID-19 pathophysiology: A review. Clin Immunol 215: 108427.

2. Ramadan N, Shaib H (2019) Middle East respiratory syndrome coronavirus (MERS-CoV): A review. Germs 9: 35-42.

3. Shereen MA, Khan S, Kazmi A, Bashir N, Siddique R, et al. (2020) COVID-19 infection: Origin, transmission, and characteristics of human coronaviruses. J Adv Res 24: 91-98.

4. Centers for Disease Control (2019) Coronavirus disease (COVID-19)cases in the US.

5. Banerjee A, Doxey AC, Mossman K, Irving AT (2021) Unraveling the Zoonotic Origin and Transmission of SARS-CoV-2. Trends Ecol Evol 36: 180-184.

6. Jayaweera M, Perera H, Gunawardana B, Manatunge J (2020) Transmission of COVID-19 virus by droplets and aerosols: A critical review on the unresolved dichotomy. Environ Res 188: 109819.

7. Li H, Liu SM, Yu XH, Tang SL, Tang CK (2020) Coronavirus disease 2019 (COVID-19): current status and future perspectives. Int J Antimicrob Agents 55: 105951.

8. Cascella M, Rajnik M, Cuomo A, Dulebohn SC, Napoli RD, et al. (2021) Features, Evaluation, and Treatment of Coronavirus (COVID-19). In: StatPearls [Internet] Treasure Island (FL).

9. Hussain A, Yadav S, Hadda V, Suri TM, Tiwari P, et al. (2020) Covid-19: a comprehensive review of a formidable foe and the road ahead. Expert Rev Respir Med 14: 869-879.

10. Junior DML, Santis GCD, Bordin JO (2020) COVID-19 convalescent plasma transfusion. Hematol Transfus Cell Ther 42: 113-115.

11. Rajendran K, Krishnasamy N, Rangarajan J, Rathinam J, Natarajan M, et al. (2020) Convalescent plasma transfusion for the treatment of COVID-19: Systematic review. J Med Virol 92: 1475-1483.

12. Xia X, Li K, Wu L, Wang Z, Zhu M, et al. (2020) Improved clinical symptoms and mortality among patients with severe or critical COVID-19 after convalescent plasma transfusion. Blood 136: 755759. 
13. Mousavizadeh L, Ghasemi S (2020) Genotype and phenotype of COVID-19: Their roles in pathogenesis. J Microbiol Immunol Infect 31: 1684-1182.

14. Polak SB, Gool ICV, Cohen D, Thüsen JHVD, Paassen JV, et al (2020) A systematic review of pathological findings in COVID-19: a pathophysiological timeline and possible mechanisms of disease progression. Mod Pathol 33: 2128-2138.

15. Huang Y, Yang C, Xu XF, Xu W, Liu SW, et al. (2020) Structural and functional properties of SARS-CoV-2 spike protein: potential antivirus drug development for COVID-19. Acta Pharmacol Sin 41: 1141-1149.

16. Shi CS, Nabar NR, Huang NN, Kehrl JH (2019) SARS-Coronavirus Open Reading Frame-8b triggers intracellular stress pathways and activates NLRP3 inflammasomes. Cell Death Discov 5: 101.

17. Li SR, Tang ZJ, Li ZH, Liu X (2020) Searching therapeutic strategy of new coronavirus pneumonia from angiotensin-converting enzyme 2: the target of COVID-19 and SARS-CoV. Eur J Clin Microbiol Infect Dis 39: 1021-1026.

18. Magrone T, Magrone M, Jirillo E (2020) Focus on Receptors for Coronaviruses with Special Reference to Angiotensin- Converting Enzyme 2 as a Potential Drug Target - A Perspective. Endocr Metab Immune Disord Drug Targets 20: 807-811.

19. Gold MS, Sehayek D, Gabrielli S, Zhang X, McCusker C, et al. (2020) COVID-19 and comorbidities: a systematic review and meta-analysis. Postgrad Med 132: 749-755.

20. Xu L, Mao Y, Chen G (2020) Risk factors for 2019 novel coronavirus disease (COVID-19) patients progressing to critical illness: a systematic review and meta-analysis. Aging 12: 12410-12421.

21. Tahvildari A, Arbabi M, Farsi $Y$, Jamshidi $P$, Hasanzadeh $S$, et al. (2020) Clinical Features, Diagnosis, and Treatment of COVID-19 in Hospitalized Patients: A Systematic Review of Case Reports and Case Series. Front Med 7: 231

22. Xie P, Ma W, Tang H, Liu D (2020) Severe COVID-19: A Review of Recent Progress with a Look toward the Future. Front Public Health 8: 189.

23. Liu MA (2019) A Comparison of Plasmid DNA and mRNA as Vaccine Technologies. Vaccines 7: 37.

24. Yang YH, Li MJ, Yi YJ, Li RF, Li CX, et al. (2021) Integrated miRNAmRNA analysis reveals the roles of miRNAs in the replanting benefit of Achyranthes bidentata roots. Sci Rep 11: 1628.

25. Abbasi J (2017) First Phase 1 Trial of a Prophylactic mRNA Vaccine Reported. JAMA 318: 2173.

26. Bialkowski L, Weijnen AV, Jeught KVD, Renmans $D$, Daszkiewicz $L$, et al. (2016) Intralymphatic mRNA vaccine induces CD8 T-cell responses that inhibit the growth of mucosally located tumours. Sci Rep 6: 22509.

27. Sebastian $M$, Papachristofilou $A$, Weiss $C$, Früh $M$, Cathomas $R$, et al. (2014) Phase Ib study evaluating a self-adjuvanted mRNA cancer vaccine (RNActive ${ }^{\circledast}$ ) combined with local radiation as consolidation and maintenance treatment for patients with stage IV non-small cell lung cancer. BMC Cancer 14: 748

28. Palucka K, Ueno H, Banchereau J (2011) Recent developments in cancer vaccines. J Immunol 186: 1325-1331.

29. Wang X, Feuerstein GZ (1997) The use of mRNA differential display for discovery of novel therapeutic targets in cardiovascular disease. Cardiovasc Res 35: 414-421.
30. Misquitta CM, lyer VR, Werstiuk ES, Grover AK (2001) The role of $3^{\prime}$-untranslated region (3'-UTR) mediated mRNA stability in cardiovascular pathophysiology. Mol Cell Biochem 224: 53-67.

31. Herman AB, Autieri MV (2018) cardiovascular disease, inflammation, and mRNA stability. Aging 10: 3046-3047.

32. mRNA vaccines: Disruptive innovation in vaccination.

33. Mai Y, Guo J, Zhao Y, Ma S, Hou Y, et al. (2020) Intranasal delivery of cationic liposome-protamine complex mRNA vaccine elicits effective anti-tumor immunity. Cell Immunol 354: 104113.

34. Pardi N, Hogan MJ, Porter FW, Weissman D (2018) mRNA vaccines a new era in vaccinology. Nat Rev Drug Discov 17: 261-279.

35. Jackson NAC, Kester KE, Casimiro D, Gurunathan S, De Rosa F, et al. (2020) The promise of mRNA vaccines: a biotech and industrial perspective. NPJ Vaccines 5: 11.

36. Zhang C, Maruggi G, Shan H, Li J (2019) Advances in mRNA Vaccines for Infectious Diseases. Front Immunol 10: 594.

37. Maruggi G, Zhang C, Li J, Ulmer JB, Yu D, et al. (2019) mRNA as a Transformative Technology for Vaccine Development to Control Infectious Diseases. Mol Ther 27: 757-772.

38. Wang F, Kream RM, Stefano GB (2020) An Evidence Based Perspective on mRNA-SARS-CoV-2 Vaccine Development. Med Sci Monit 26: 924700.

39. Kowalski PS, Rudra A, Miao L, Anderson DG (2019) Delivering the Messenger: Advances in Technologies for Therapeutic mRNA Delivery. Mol Ther 27: 710-728.

40. Wadhwa A, Aljabbari A, Lokras A, Foged C, Thakur A, et al. (2020) Opportunities and Challenges in the Delivery of mRNA-based Vaccines. Pharmaceutics 12: 102.

41. Gómez-Aguadol, Rodríguez-Castejón J, Vicente-Pascual $M$, Rodríguez-Gascón A, Solinís MÁ, et al. (2020) Nanomedicines to Deliver mRNA: State of the Art and Future Perspectives. Nanomaterials (Basel) 10: 364

42. Schlake T, Thess A, Fotin-Mleczek M, Kallen KJ (2012) DevelopingmRNA-vaccinetechnologies. RNA Biol 9: 1319-1330.

43. Reichmuth $A M$, Oberli $M A$, Jaklenec $A$, Langer $R$, Blankschtein $D$ (2016) mRNA vaccine delivery using lipid nanoparticles. Ther Deliv 7: 319- 334

44. Midoux P, Pichon C (2015) Lipid-based mRNA vaccine delivery systems. Expert Rev Vaccines 14: 221-234.

45. Plotkin SA, Wang D, Oualim A, Diamond DJ, Kotton CN, et al. (2020) The status of vaccine development against the human cytomegalovirus. J Infect Dis 221: S113-S122.

46. Lindsay KE, Bhosle SM, Zurla C, Beyersdorf J, Rogers KA, et al. (2019) Visualization of early events in mRNA vaccine delivery in non-human primates via pet-CT and near-infrared imaging. Nat Biomed Eng 3: 371-380.

47. Geall AJ, Verma A, Otten GR, Shaw CA, Hekele A, et al. (2012) Nonviral delivery of self-amplifying RNA vaccines. Proc Natl Acad Sci U S A 109: 14604-14609.

48. Maruggi G, Chiarot E, Giovani C, Buccato S, Bonacci S, et al. (2017) Immunogenicity and protective efficacy induced by self-amplifying mRNA vaccines encoding bacterial antigens. Vaccine 35: 361-368.

49. Huysmans H, Zhong Z, De Temmerman J, Mui BL, Tam YK, et al. (2019) Expression kinetics and innate immune response after electroporation and Inp-mediated delivery of a self-amplifying mRNA in the skin. Mol Ther Nucleic Acids 17: 867-878. 
50. Brito LA, Kommareddy S, Maione D, Uematsu Y, Giovani C, et al. (2015) Self-amplifying mRNA vaccines. Adv Genet 89: 179-233.

51. Laube BL (2014) The expanding role of aerosols in systemic drug delivery, gene therapy and vaccination: An update. Transl Respir Med 2: 3.

52. Hellfritzsch $M$, Scherließ $R$ (2019) Mucosal vaccination via the respiratory tract. Pharmaceutics 11: 375.

53. Ogra PL, Faden H, Welliver RC (2001) Vaccination strategies for mucosal immune responses. Clin Microbiol Rev 14: 430-445.

54. Cesta MF (2006) Normal structure, function, and histology of mucosa-associated lymphoid tissue. Toxicol Pathol 34: 599-608.

55. Wu HY, Nguyen HH, Russell MW (1997) Nasal lymphoid tissue (NALT) as a mucosal immune inductive site. Scand J Immunology 46: 506513.

56. Date Y, Ebisawa M, Fukuda S, Shima H, Obata Y, et al. (2017) NALT M cells are important for immune induction for the common mucosal immune system. Int Immunol 29: 471-478.

57. Li M, Zhao M, Fu Y, Li Y, Gong T, et al. (2016) Enhanced intranasal delivery of mRNA vaccine by overcoming the nasal epithelial barrier via intra- and paracellular pathways. J Control Release 228: 9-19.

58. Danaei M, Dehghankhold M, Ataei S, Davarani FH, Javanmard R, et al. (2018) Impact of particle size and polydispersity index on the clinical applications of lipidic nanocarrier systems. Pharmaceutics 10: 57

59. Xu J, Wong DH, Byrne JD, Chen K, Bowerman C, et al. (2013) Future of the particle replication in nonwetting templates (PRINT) technology Angew Chem Int Ed Engl 52: 6580-6589.

60. Sharma S, Mukkur TK, Benson HA, Chen Y (2009) Pharmaceutical aspects of intranasal delivery of vaccines using particulate systems. J Pharm Sci 98: 812-843.

61. Yang W, Peters JI, Williams RO $3^{\text {rd }}(2008)$ Inhaled nanoparticles--a current review. Int J Pharm 356: 239-247.

62. Paranjpe M, Muller-Goymann CC (2014) Nanoparticle-mediated pulmonary drug delivery: A review. Int J Mol Sci 15: 5852-5873.

63. Griffin DE (2014) Current progress in pulmonary delivery of measles vaccine. Expert Rev Vaccines 13: 751-759.

64. Elhissi A (2017) Liposomes for pulmonary drug delivery: The role of formulation and inhalation device design. Curr Pharm Des 23: 362 372.

65. Omri A (2015) Pulmonary drug and vaccine delivery: Therapeutic significance and major challenges. Expert Opin Drug Deliv 12: 853855.

66. Fan YN, Li M, Luo YL, Chen Q, Wang L, et al. (2018) Cationic lipidassisted nanoparticles for delivery of mRNA cancer vaccine. Biomater Sci 6: 3009-3018.
67. Espeseth AS, Cejas PJ, Citron MP, Wang D, DiStefano DJ, et al. (2020) Modified mRNA/lipid nanoparticle-based vaccines expressing respiratory syncytial virus $F$ protein variants are immunogenic and protective in rodent models of RSV infection. NPJ Vaccines 5: 16.

68. Feldman RA, Fuhr R, Smolenov I, Mick Ribeiro A, Panther L, et al. (2019) mRNA vaccines against H10N8 and H7N9 influenza viruses of pandemic potential are immunogenic and well tolerated in healthy adults in phase 1 randomized clinical trials. Vaccine 37: 3326-3334.

69. Bahl K, Senn JJ, Yuzhakov O, Bulychev A, Brito LA, et al. (2017) Preclinical and clinical demonstration of immunogenicity by mRNA vaccines against H1ON8 and H7N9 influenza viruses. Mol Ther 25 1316-1327.

70. Moderna (2020) Moderna advances late-stage development of its vaccine (mRNA-1273) against COVID-19.

71. Inovio (2021) Rapid creation of a novel, investigational Covid-19 DNA vaccine.

72. Clinical Trails Arena (2020) Oxford Covid-19 vaccine trials start in south Africa and Brazil.

73. Pfizer (2021) Biontech and pfizer announce completion of dosing for first cohort of phase $1 / 2$ trial of Covid-19 vaccine candidates in Germany.

74. Johnson \& Johnson (2021) Johnson \& Johnson announces a lead vaccine candidate for COVID-19; landmark new partnership with U.S. Department of Health \& Human Services; and commitment to supply one billion vaccines worldwide for emergency pandemic use.

75. (2021) UQ COVID-19 vaccine shown to induce potent protective response in pre-clinical trials. The University of Queenslands, Australia.

76. Altimmune. Pipeline for intranasal vaccine development.

77. Novavax (2020) Novavax initiates phase $1 / 2$ clinical trial of COVID-19 vaccine.

78. Biospace (2021)Vaxart plans to push COVID-19 vaccine candidate into the clinic

79. Baden LR, El Sahly HM, Essink B, Kotloff K, Frey S, et al. (2020) Efficacy and safety of the mRNA-1273 SARS-CoV-2 vaccine. N Engl J Med Epub 384: 403-416.

80. Polack FP, Thomas SJ, Kitchin N, Absalon J, Gurtman A, et al. (2020) Safety and efficacy of the BNT162b2 mRNA Covid-19 vaccine. N Eng J Med 383: 2603-2615.

81. Randolph HE, Barreiro LB (2020) Herd immunity: Understanding COVID-19. Immunity 52: 737-741.

82. de Alwis R, Chen S, Gan ES, Ooi EE (2020) Impact of immune enhancement on Covid-19 polyclonal hyperimmune globulin therapy and vaccine development. EBioMedicine 55: 102768.

83. Ayoub BM (2020) COVID-19 vaccination clinical trials should consider multiple doses of BCG. Pharmazie 75: 159 